\title{
Diagnostic implications of MOG/AQP4 antibodies in recurrent optic neuritis
}

\author{
YING PENG $^{1}$, LEI LIU ${ }^{2}$, YUANCHU ZHENG ${ }^{2}$, ZHIXIN QIAO $^{3}$, KAI FENG $^{1}$ and JIAWEI WANG ${ }^{2,3}$ \\ ${ }^{1}$ Department of Neurology, Shunyi District Hospital, Beijing 101300; ${ }^{2}$ Department of Neurology; \\ ${ }^{3}$ Central Laboratory, Beijing Tongren Hospital, Capital Medical University, Beijing 100010, P.R. China
}

Received November 10, 2017; Accepted April 6, 2018

DOI: $10.3892 /$ etm.2018.6273

\begin{abstract}
The present study aimed to detect myelin oligodendrocyte glycoprotein (MOG) and aquaporin-4 (AQP4) antibodies in serum specimens of patients with recurrent optic neuritis (RON) through establishing 293 cells with stable expression of MOG and the complete genomic sequence as the substrate using a cell-based assay (CBA). Furthermore, the clinical features of MOG antibody-positive recurrent optic neuritis (MOG-RON) were assessed. A total of 43 RON patients admitted to Beijing Tongren Hospital from December 2014 to May 2015 were enrolled, including 11 males and 32 females. The serum was collected from all patients, and the MOG and AQP4 antibodies were detected via the CBA. According to the results, the 43 patients were divided into four groups, namely the MOG antibody-positive group $(n=11)$, the AQP4 antibody-positive group $(n=20)$, the MOG/AQP4 antibody-positive group $(n=1)$ and the MOG/AQP4 antibody-negative group $(n=11)$. Clinical data were collected and all patients were followed up for 6 months, with parameters observed including the visual acuity, visual field and ocular fundus. The differences in the demographics, clinical features, characteristics of imaging examination, vision at onset and visual function recovery at 6 months after treatment were compared among the different groups. The characteristics of MOG antibody-positive RON were summarized. Of the 43 RON patients, $2.33 \%$ was both MOG and AQP4 antibody-positive, $27.91 \%$ were MOG antibody-positive. Compared with the AQP4-RON patients, there were relatively less MOG-RON patients (63.6 vs. 95.0\%) and the canal segment and intracranial segment of the optic canal were less involved $(\mathrm{P}<0.05)$. The visual acuity at onset of MOG-RON was not inferior to that of AQP4-RON, and the
\end{abstract}

Correspondence to: Dr Jiawei Wang, Department of Neurology, Beijing Tongren Hospital, Capital Medical University, 1 Dongjiaomin Alley, Dongcheng, Beijing 100010, P.R. China E-mail: pengying1991@126.com

Key words: myelin oligodendrocyte glycoprotein antibody, aquaporin-4 antibody, with recurrent optic neuritis, neuromyelitis optica spectrum disorders visual recovery degree of MOG-RON was better $(\mathrm{P}<0.05)$. MOG antibody may be detected in the serum of certain RON patients, which have unique and different characteristics from AQP4 antibody-positive RON patients, so it may be used as a prognostic biomarker for RON. Furthermore, MOG antibody is present in the serum of patients with neuromyelitis optica spectrum disorders and may be a potential biomarker for these conditions.

\section{Introduction}

Optic neuritis $(\mathrm{ON})$ refers to a spectrum of inflammatory lesions involving the optic nerve; it is a blinding optic nerve disease that occurs most frequently in young and middle-aged individuals. The most common type is idiopathic $\mathrm{ON}$, which is further subdivided into idiopathic demyelinating optic neuritis (IDON), also known as multiple sclerosis-associated optic neuritis (MS-ON), neuromyelitis optica-associated optic neuritis (NMO-ON) and $\mathrm{ON}$ associated with other central nervous system demyelinating diseases (1). NMO-ON has a higher disability rate compared with IDON, and an aquaporin-4 (AQP4) immunoglobulin $\mathrm{G}(\mathrm{IgG})(+)$ status has a decisive role in the diagnosis of NMO-ON. The probability of progressing to NMO-ON in AQP4 IgG (+) patients is significantly higher than that of AQP4 IgG (-) patients, and the rate of progression to NMO-ON is faster in AQP4 IgG (+) patients. Detection of AQP4 IgG, as a clinical biomarker for identifying NMO-ON and other demyelinating diseases, is helpful for the early diagnosis and prognostication of NMO-ON patients. However, a clinical study indicated that $10-20 \%$ of NMO-ON patients have an AQP4 IgG (-) status (2).

New diagnostic criteria for $\mathrm{NMO}-\mathrm{ON}$ were developed by the International NMO Diagnostic Group in 2015 (3); diagnostic criteria for serum-negative NMO-ON were proposed, and it was suggested to actively search for potential alternative biomarkers for AQP4 IgG (-) NMO-ON patients. It has been reported that AQP4 IgG (-) NMO-ON patients are myelin oligodendrocyte glycoprotein (MOG) $\operatorname{IgG}(+)(4,5)$, and it is therefore speculated that MOG $\mathrm{IgG}$ is involved in the pathogenesis in these serum AQP4 IgG (-) NMO-ON patients. Saadoun et al (6) microinjected MOG IgG, sourced from patients with NMO, into mouse brains and compared the results with those obtained by AQP4 IgG injection. The results indicated that MOG IgG caused myelin changes 
and altered the expression of axonal proteins within two weeks, but did not produce any inflammation, axonal loss or neuronal or astrocyte death, while AQP4 IgG produced complement-mediated myelin loss, and neuronal and astrocyte death with limited recovery at two weeks. In addition, MOG $\mathrm{IgG}$ is also detected in the serum of certain adolescent patients with acute disseminated encephalomyelitis and multiple sclerosis; besides intracranial lesions, these patients also suffer from ON. Therefore, MOG IgG should be actively screened for ON patients with an unknown cause.

\section{Patients and methods}

Patients. A total of 43 RON patients (11 males and 32 females; age, 30-51 years) admitted to the Neurology Department of Beijing Tongren Hospital Affiliated to Capital Medical University (Beijing, China) from December 2014 to May 2015 were enrolled in the present study. At the same time, 8 healthy individuals (4 males and 4 females; age, 32-49 years) admitted to the same hospital from December 2014 to May 2015 were included randomly. The present study was approved by the ethics committee of Beijing Tongren Hospital (Beijing, China). Written informed consent was obtained from the patients and/or their guardians, as well as the healthy individuals.

Inclusion/exclusion criteria. Patients with an acute ON attack meeting the diagnostic criteria of the American Optic Neuritis Study Group (1) were included. They were required to have had two or more $\mathrm{ON}$ attacks previously according to their complete medical data. Patients with contraindications for intravenous application of methylprednisolone and/or immunosuppressors, and patients with other ophthalmic diseases (including anterior segment lesions, vitreous lesions, retinopathy, refractive errors and glaucoma), other types of ON (including ischemic, oppressive, invasive, traumatic, toxic, nutritional metabolic and hereditary disease), diminution of vision due to intracranial diseases (e.g., other cerebrovascular, infectious, traumatic, degenerative, genetic metabolic or nutritional metabolic disease, or poisoning), alcohol encephalopathy or Alzheimer's disease, as well as pregnant or lactating patients were excluded from the study.

Diagnostic criteria for ON. The diagnosis of ON was based on the diagnostic criteria of the American Optic Neuritis Study Group (1): Acute vision loss accompanied with or without eye pain, nerve fibre bundle damage-associated visual field anomaly and at least one of the following two criteria: Relative afferent pupillary defect and visual evoked potential abnormality; no clinical and laboratory evidence of compressive, ischemic, toxic, hereditary, metabolic and invasive optic neuropathy; clinical and laboratory evidence of retinal disease and other ocular and neurological disorders without leading to acute vision loss; patients with typical onset of acute ON for $>24 \mathrm{~h}$ via objective examination with an interval of at least one month since the last episode.

Data collection. The demographic data (onset age and sex) and clinical features, including the course of the disease, monocular/binocular involvement, eye pain, optic disc edema, worst visual acuity at onset, worst videofluoroscopic swallowing study (VFSS) at onset, visual recovery after treatment with prednisone and/or immunosuppressive agents, VFSS score after treatment, onset frequency of $\mathrm{ON}$, involvement of intraorbital segment, canal segment and intracranial segment of optic nerve and optic chiasma, the presence of other lesions involving the central nervous system, other autoimmune abnormalities (e.g., anti-nuclear antibody, anti-extractable nuclear antigens antibody spectrum, anti-double-stranded DNA antibody, human leukocyte antigen-B27, anti-neutrophil cytoplasmic antibodies and cardiolipin antibody) of patients were collected. AQP4 and MOG IgG in the serum of patients were detected via the cell-based assay (CBA). All patients were treated with methylprednisolone in the acute phase, and certain patients received the immunosuppressive therapy during the process of hormone reduction. One patient was diagnosed with multiple sclerosis and treated with interferon. All patients were followed up, and the visional recovery of patients at 6 months after the last onset was recorded via outpatient review upon visitation to the clinc.

CBA for detection of AQP4 and MOG IgG status. The 293 cell line (widely known as Human Embryonic Kidney 293 cells) was obtained from the Cell Bank of the Chinese Academy of Sciences (Shanghai, China). The 293 cells with stable expression of MOG were established as the substrate for the CBA. AQP4 IgG and MOG IgG in the serum specimens of recurrent $\mathrm{ON}$ (RON) patients was detected via indirect immunofluorescence assay (4). The expression vectors of the full-length sequences of AQP4 and MOG were constructed, and plasmids were transferred into 293 cells with the Neofect transfection reagent (Nofectbiotech, Beijing, China). At $24 \mathrm{~h}$ after transfection, the cells were fixed with immune fixative (4\% Polyoxymethylene; Sigma-Aldrich; Merck KGaA, Darmstadt, Germany) and blocked with $10 \%$ goat serum (Gibco; Thermo Fisher Scientific, Inc., Waltham, MA, USA) for $30 \mathrm{~min}$. Patient serum was diluted at 1:100 and incubated with 293 cells at room temperature overnight. The cells were incubated with Alexa Fluor 488-conjugated sheep anti-human antibody (1:250; cat. no. ab201540; Abcam, Cambridge, $\mathrm{UK}$ ) for $30 \mathrm{~min}$ at $20^{\circ} \mathrm{C}$, and then washed three times with PBS containing Tween-20, followed by staining with DAPI $(0.1 \mathrm{ug} / \mathrm{ml})$ for $10 \mathrm{~min}$ at $20^{\circ} \mathrm{C}$. The cells were then observed under a fluorescence microscope after mounting. The AQP4 and MOG IgG status of RON patients was then determined; AQP4 and MOG are transmembrane glycoproteins, and NMO-associated IgGs are marked by green fluorescence in the cell membrane. A positive and negative control were set up in the experiment as follows: Rabbit anti-human MOG (1 $\mu \mathrm{g} / \mathrm{ml}$; cat. no. M0695) and AQP4 $(1 \mu \mathrm{g} / \mathrm{ml})$ antibody (cat. no. G9626; both Sigma-Aldrich; Merck KGaA) was added to the serum obtained from a healthy individual as the positive control at room temperature overnight, while serum obtained from a healthy individual was used as a negative control.

RON grouping. AQP4 antibody and MOG IgG in patients meeting the diagnostic criteria for RON were detected via the CBA. According to the results, they were divided into MOG IgG (+) AQP4 IgG (-) group [MOG (+) group], MOG IgG (-) AQP4 IgG (+) group [AQP4 IgG (+) group], MOG IgG (-) and AQP4 IgG (-) group [MOG/AQP4 IgG (-) group] and MOG 
A

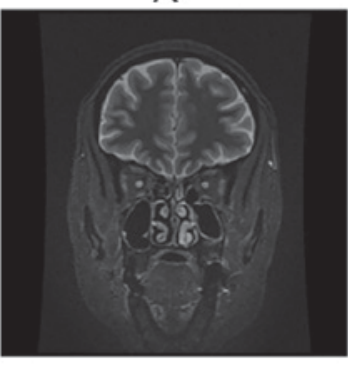

E
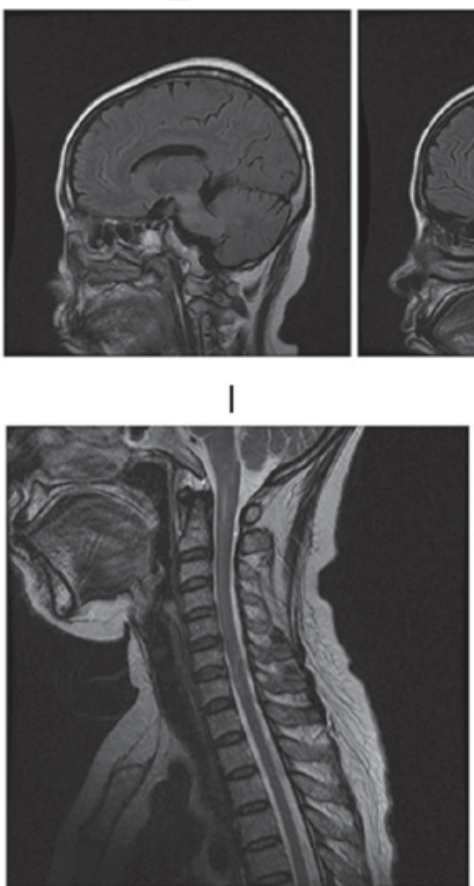

$\mathrm{B}$

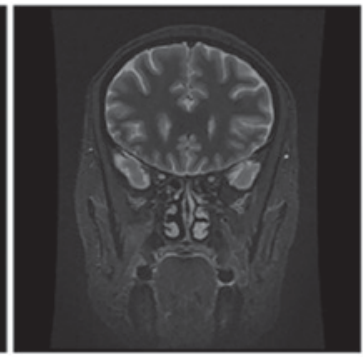

$\mathrm{F}$

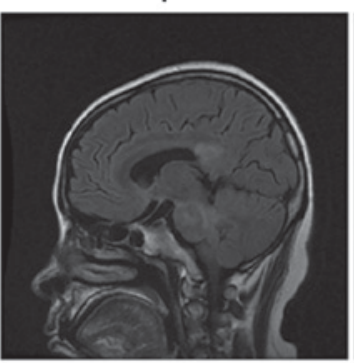

J
C

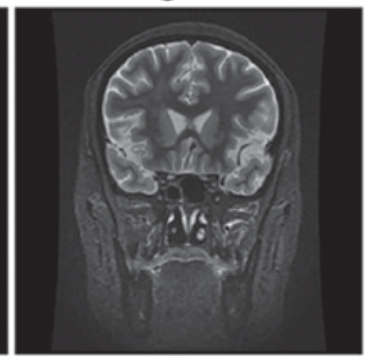

G

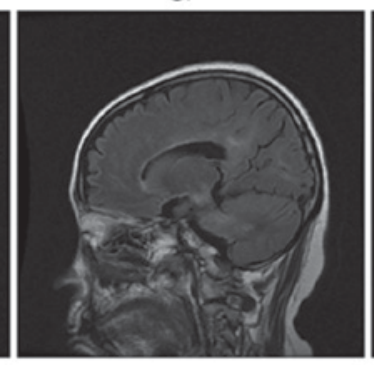

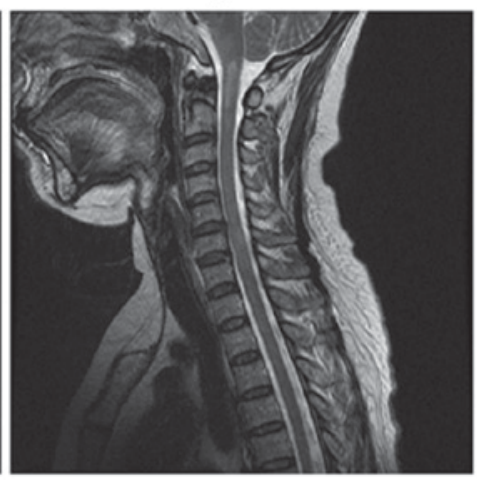

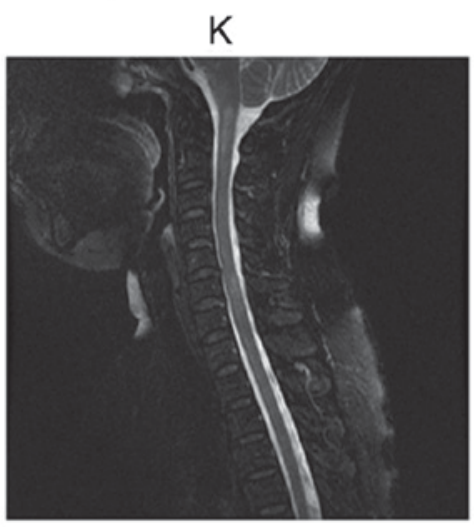

D

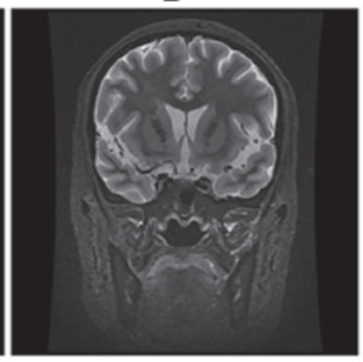

$\mathrm{H}$

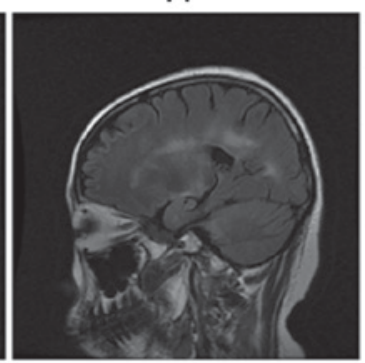

$\mathrm{K}$

Figure 1. A female myelin oligodendrocyte glycoprotein antibody(+) and aquaporin-4 antibody(-) neuromyelitis optica-associated ON patient with an onset age of 38 years, disease duration of 16 years and a total of 15 recurrent attacks presented with an attack of ON on both eyes, acute myelitis involving $>3$ segments, postrema syndrome and acute diencephalic syndrome. (A-D) The brain MRI coronal short-time inversion recovery revealed that the intraorbital segment, canal segment and intracranial segment of optic nerve and optic chiasma became thinner and the signal was increased when compared with an earlier image obtained from patients prior to the present study. (E-H) The brain MRI sagittal T2 fluid-attenuated inversion recovery revealed the abnormal signal in bilateral basal ganglia, thalamus, lateral ventricle, brain stem and corpus callosum. (I-K) Cervical MRI sagittal T2-weighted images revealed the abnormal signal at the medullary-C2 and C5-T1. ON, optic neuritis; MRI, magnetic resonance image.

IgG (+) and AQP4 IgG (+) group [MOG/AQP4 IgG (+) group]. The demographic data, degree of visual impairment, brain and optic nerve magnetic resonance imaging (MRI) and other laboratory test data were collected. The clinical characteristics of each subgroup were analyzed.

Observational indexes. The visual function data of the 43 patients enrolled were collected and the visual impairment of 83 affected eyes was observed. A corrected visual acuity of the affected eye of $>0.1$ indicated relatively good visual acuity, while that of $\leq 0.1$ indicated poor visual acuity. Visual function VFSS score were determined prior to and at 6 months after treatment with methylprednisolone and/or immunosuppressive agents.

Statistical analysis. Statistical analysis was performed using SPSS 18.0 software (SPSS, Inc., Chicago, IL, USA). A normality test was performed for continuous variables; data with a normal distribution were expressed as the mean \pm standard deviation, while those with a skewed distribution were expressed as the median [25\% quartile-75\% quartile (Q25-Q75)]. One-way analysis of variance was used for intergroup comparisons, followed by the Least-Significant Difference test as a post-hoc test, while the Kruskal-Wallis test followed by a Mann-Whitney $\mathrm{U}$ post-hoc test with Bonferroni's correction was applied in the case of an abnormal distribution. The chi-square test was used for categorical variables. The demographics, clinical features, characteristics of laboratory and imaging examinations, vision at onset and visual function recovery at 6 months after treatment were compared among the MOG IgG (+) group, AQP4 IgG (+) group, MOG/AQP4 IgG (+) and MOG/AQP4 IgG (-) groups. $\mathrm{P}<0.05$ was considered to indicate a statistically significant difference.

\section{Results}

Case presentation of a NMO-ON patient with MOG IgG (+) status. Fig. 1 displays representative MRI images of a female MOG IgG (+) NMO-ON patient with the onset age of 38 years, 


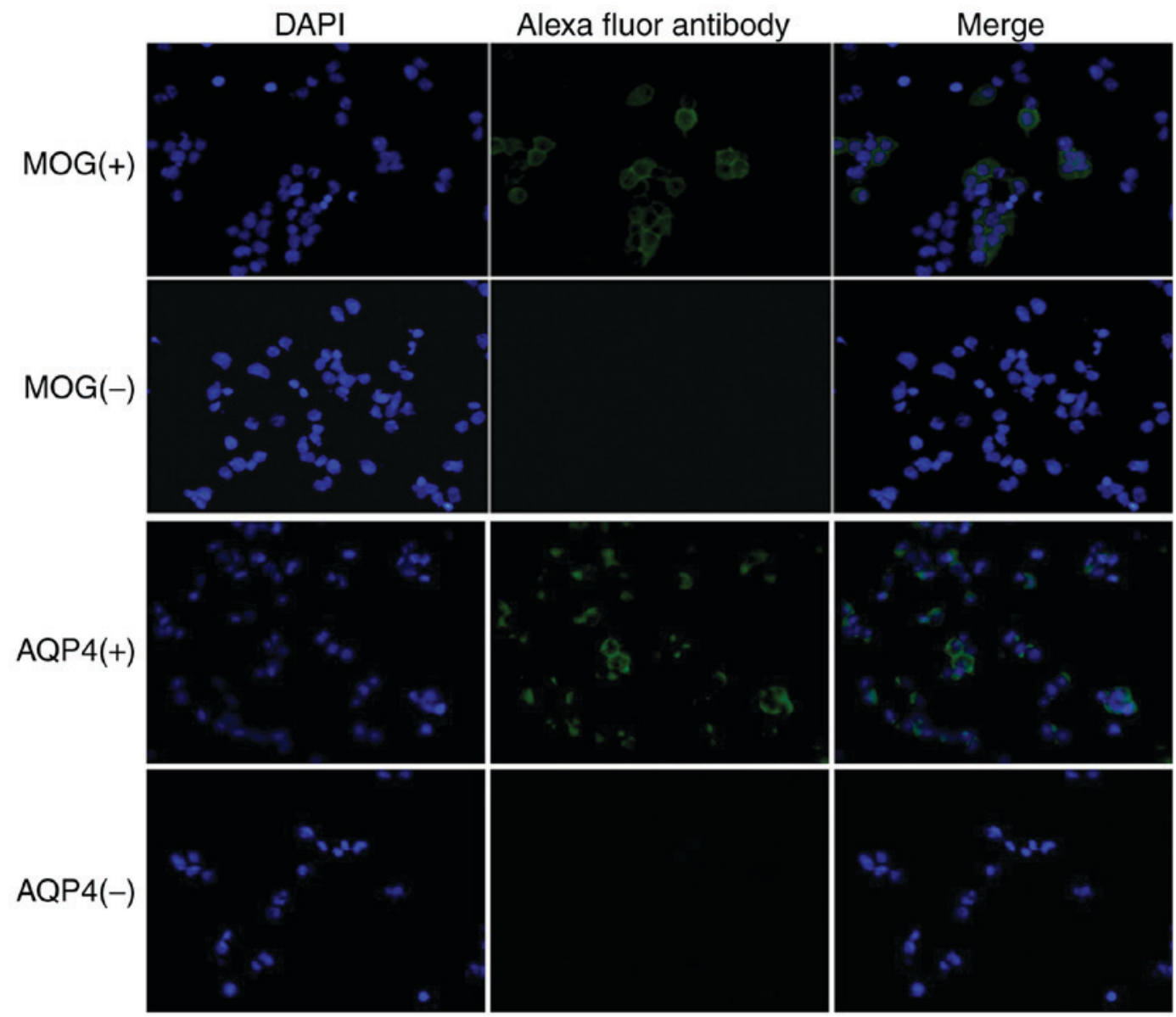

Figure 2. MOG antibody was detected by a cell-based assay (magnification, x100). 293 cells were observed under a fluorescence microscope. Blue fluorescence represents nuclei and green fluorescence represents Alexa fluor 488-conjugated MOG antibody. MOG, myelin oligodendrocyte glycoprotein; AQP, aquaporin.

course of disease of 16 years and a history of 15 attacks who had acute $\mathrm{ON}$ in both eyes. The patient presented with acute myelitis involving $>3$ segments, postrema syndrome and acute diencephalic syndrome. As presented in Fig. 1A-D, the brain MRI coronal short-time inversion recovery revealed that the intraorbital segment, canal segment and intracranial segment of the optic nerve and optic chiasma had become thinner and the signal was increased when compared with earlier images obtained from patients prior to the study. As presented in Fig. 1E-H, the brain MRI sagittal T2 fluid-attenuated inversion recovery revealed an abnormal signal in the bilateral basal ganglia, thalamus, lateral ventricle, brain stem and corpus callosum. As presented in Fig. 1I-K, cervical MRI sagittal T2-weighted images revealed an abnormal signal in medullary-C2 and C5-T1.

Detection of MOG IgG via CBA. The 293 cells with stable expression of the complete genomic sequence of MOG were successfully constructed. MOG IgG was detected via a CBA and observed under a fluorescence microscope. As presented in Fig. 2, the representative images demonstrated that the cells incubated with serum from the MOG IgG (+) and AQP4 (+) patients had green fluorescence while DAPI-stained nuclei exhibited blue fluorescence.

Basic data and clinical features of NMO-ON patients in each group. A total of 43 patients hospitalized from December 2014 to May 2015 met the inclusion criteria. The cohort comprised 11 cases of MOG IgG (+) (25.58\%), 20 cases of AQP4 IgG (+) (46.51\%), 11 cases of MOG/AQP4 IgG (-) (25.58\%) and 1 case of MOG/AQP4 IgG (+) (2.33\%; Fig. 3). A total of 32 females $(74.42 \%)$ and 11 males $(25.58 \%)$ with an average age at onset of $33 \pm 12$ years and the total course of disease of 24 months (Q25-Q75, 12-111) were included. A total of 27 cases $(62.79 \%)$ had eye pain and 17 cases $(39.53 \%)$ presented with optic disk edema after onset. In the 43 patients, 83 eyes were affected, including 20 eyes in the MOG IgG (+) group, 39 eyes in the AQP4 IgG (+) group, 22 eyes in the MOG/AQP4 IgG (-) group and 2 eyes in the MOG/AQP4 IgG (+) group. The brain MRI indicated that there were 15 cases $(34.88 \%)$ of demyelinating lesions in the brain parenchyma, and optic nerve MRI revealed that there were 43 cases $(100.00 \%)$ with intraorbital segment involvement of the optic nerve, 32 cases $(74.42 \%)$ with canal segment involvement, 18 cases $(18.60 \%)$ with intracranial segment involvement and 8 cases (18.60\%) with optic chiasma involvement. Furthermore, the spinal MRI confirmed that there were 5 cases $(11.62 \%)$ of spinal cord involvement, including 4 cases $(9.30 \%)$ of spinal cord involvement of $\geq 3$ segments. Serological examination indicated that 18 cases $(41.86 \%)$ had abnormalities in other autoimmune indexes.

As presented in Tables I and II, the AQP4 IgG (+) group was mostly comprised of females compared with the MOG 


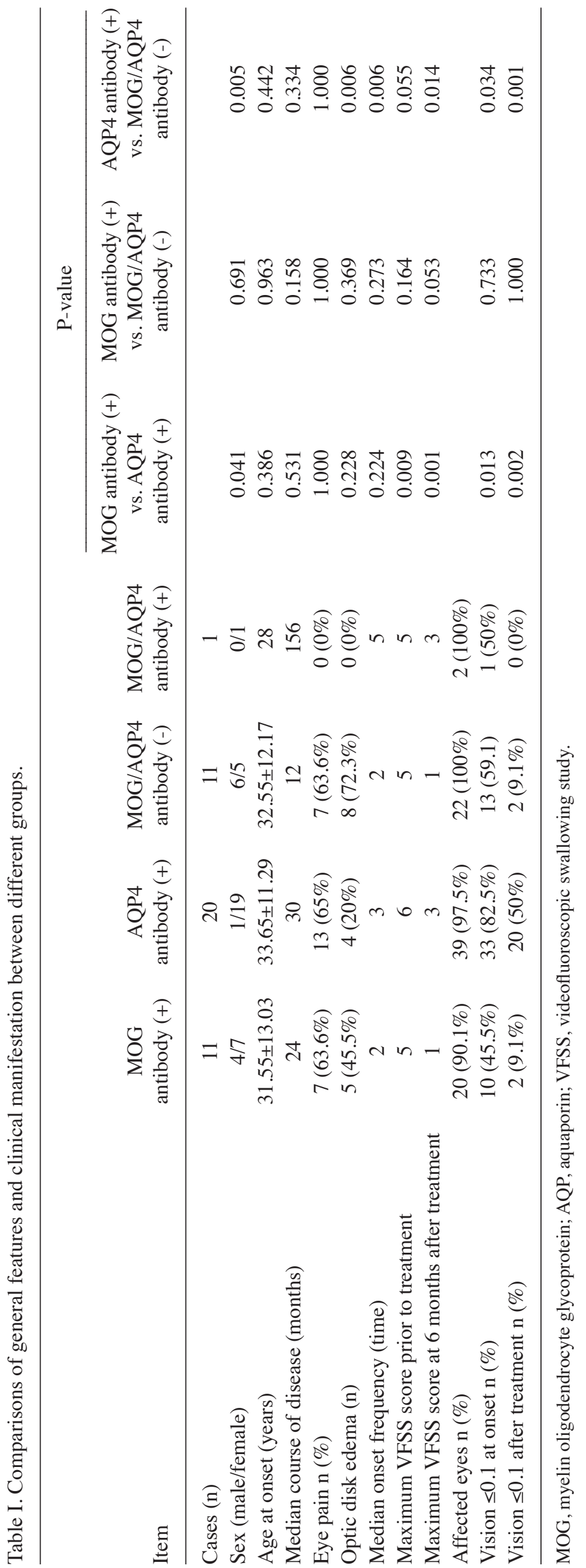




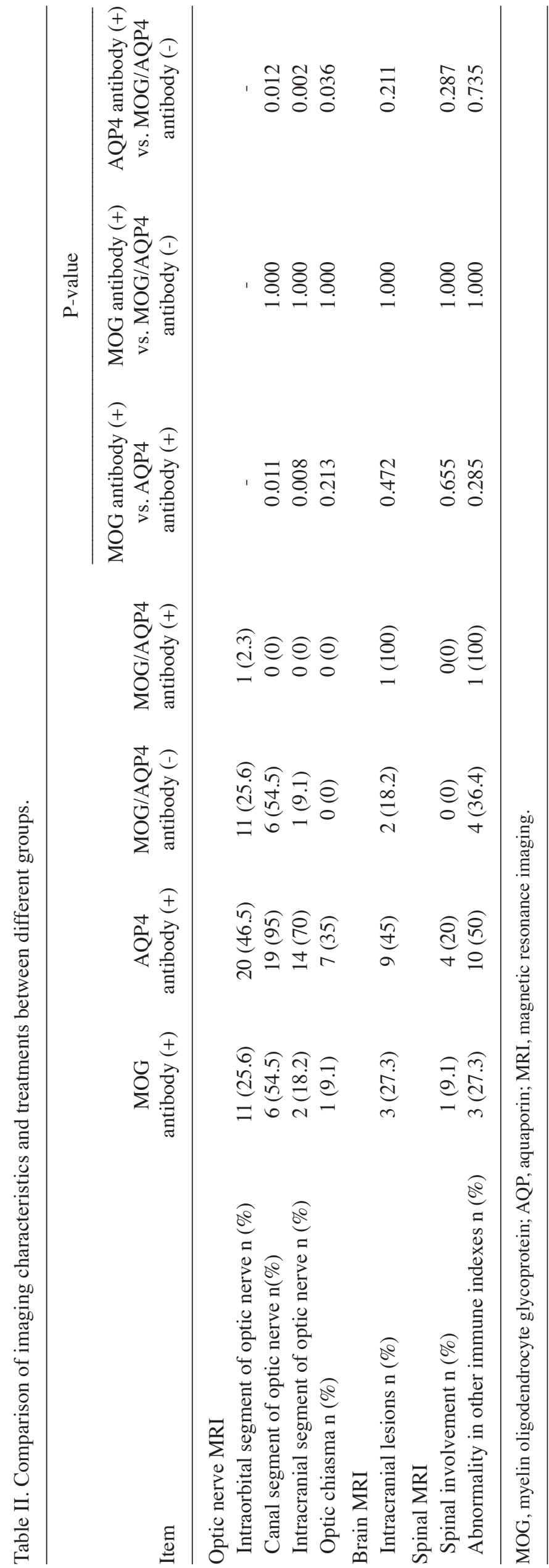




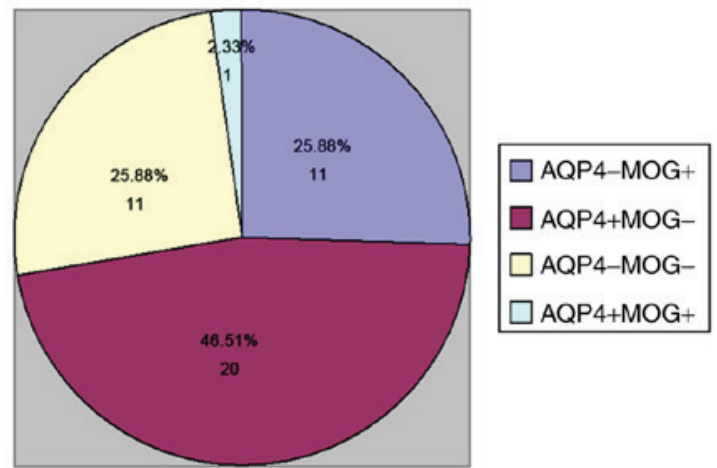

Figure 3. Percentage of AQP4 antibody (+) and MOG antibody (+) patients among recurrent optic neuritis patients. MOG, myelin oligodendrocyte glycoprotein; AQP, aquaporin.

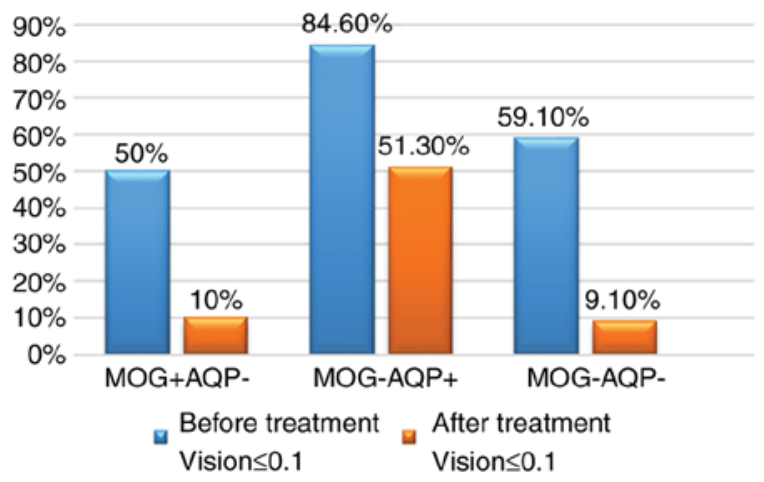

Figure 4. Percentage of patients with a vision of $\leq 0.1$ in the different groups prior to and after treatment. MOG, myelin oligodendrocyte glycoprotein; $\mathrm{AQP}$, aquaporin.

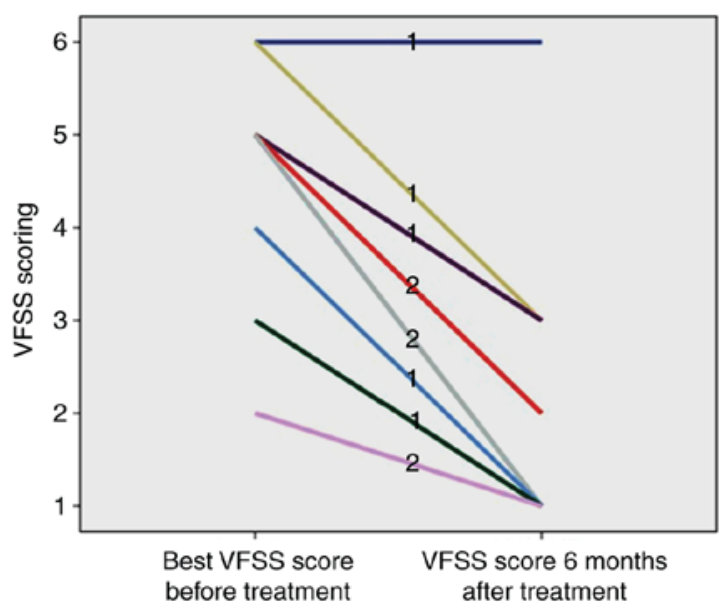

Figure 5. The VFSS score of myelin oligodendrocyte glycoprotein antibody (+) recurrent optic neuritis patients prior to and at 6 months after treatment. VFSS, videofluoroscopic swallowing study.

IgG (+) group and the MOG/AQP4 IgG (-) group $(\mathrm{P}<0.05)$. Compared with the MOG/AQP4 IgG (-) group, optic disk edema was rarer with fewer attacks in the AQP4 $\mathrm{IgG}(+)$ group $(\mathrm{P}<0.05)$; however, no statistically significant difference was identified between the MOG IgG (+) and the AQP4 IgG (+) and MOG/AQP4 IgG (-) groups. Optic nerve MRI indicated that the intraorbital segments of the optic nerve were involved in the three groups, but the canal segment and intracranial segment of the optic nerve in the AQP4 IgG (+) group were more significantly involved than those in the other two groups $(\mathrm{P}<0.05)$. The optic chiasma was more significantly involved in the AQP4 IgG (+) group than that in the MOG/AQP4 $\operatorname{IgG}(-)$ group $(\mathrm{P}<0.05)$, and there was no statistically significant difference in the involvement of optic chiasma between the MOG IgG (+) group and the other two groups. There were no statistically significant differences in the age at onset, occurrence of eye pain, course of disease, intracranial lesions, spinal cord involvement and other immune indexes among the three groups.

Comparison of visual function prognosis of patients. All patients enrolled were followed up at the clinic 6 months after onset, with items assessed including visual acuity, visual field and ocular fundus. All of the patients received the same treatment. Prior to treatment, poor visual acuity was determined in 10 eyes $(50.0 \%)$ of the MOG IgG (+) group, 33 eyes $(84.6 \%)$ of the AQP4 IgG (+) group and 13 eyes $(59.1 \%)$ of the MOG/AQP4 IgG (-) group. At 6 months after treatment, poor visual acuity was still present in 2 eyes $(10.0 \%)$ of the MOG $\operatorname{IgG}(+), 20$ eyes (51.3\%) of the AQP4 $\operatorname{IgG}(+)$ and 2 eyes $(9.1 \%)$ of the MOG/AQP4 IgG (-) group (Fig. 4). The vision at onset was worse and the recovery was poorer in the AQP4 $\operatorname{IgG}(+)$ group compared with those in the MOG IgG (+) group and MOG/AQP4 IgG (-) groups ( $\mathrm{P}<0.05$; Table I).

As presented in Table I, the VFSS score was relatively lower with the worst visual function after onset and the visual function recovery after treatment was better in the MOG $\mathrm{IgG}(+)$ group compared with that in the AQP4 $\operatorname{IgG}(+)$ group $(\mathrm{P}<0.05)$. As presented in Figs. 5 and 6, although 1 case in the MOG IgG (+) group (9.09\%) had a poor recovery of visual function, the remaining 10 cases $(90.91 \%)$ had a better recovery of visual function, and the VFSS score after treatment ranged between 1 and 3. The majority of patients in the AQP4 IgG (+) group (14 cases, $70.00 \%$ ) had a poor visual function recovery. After treatment, the VFSS score ranged between 4 and 6; a small number of AQP4 IgG (+) patients (6 cases, 30.00\%) had a better recovery, and the score ranged between 1 and 3 .

Association between MOG IgG and NMO-ON. Out of the 12 MOG IgG (+) patients, 2 met the diagnostic criteria for NMO-ON (Table I) (2), including 1 case of AQP4 IgG (+) NMO-ON; this patient had a course of disease of 13 years, a right vision at onset of $\leq 0.1$ and a left vision at onset of $>0.1$, and presented with a recurrent attack of $\mathrm{ON}$ in both eyes. After hormone and immunosuppressive therapy, the vision was recovered to be $>0.1$ in both eyes, and the VFSS score was recovered from 5 to 3 points; the intraorbital segment of the optic nerve was involved, but the intracranial segment, canal segment and optic chiasma were not involved; there were demyelinating lesions in the intracranial semi-oval center, but the spinal cord was not involved. The other case as aforementioned was an AQP4 IgG (-) NMO-ON patient with a course of disease of 16 years and a history of 15 attacks, who presented with a recurrent attack of ON in both eyes, acute myelitis involving $>3$ segments, postrema syndrome and acute diencephalic syndrome, which used to be diagnosed as 'multiple sclerosis'. After interferon treatment, the patient 
was diagnosed with NMO-ON and treated with hormone and immunosuppressive therapy. The recovery of vision on both eyes was poor $(\leq 0.1)$; The VFSS score prior to and after treatment was 6 points. Imaging indicated involvement of the intraorbital segment, canal segment, intracranial segment and optic chiasma of bilateral optic nerves, demyelinating lesions in the intracranial medulla oblongata, thalamus, midbrain, corpus callosum, basal ganglia, frontal white matter, lateral ventricle, and medullary C2 and C5-T1.

\section{Discussion}

In the present study, $27.91 \%$ of RON patients were MOG $\operatorname{IgG}(+)$ [including 1 case that was also AQP4 $\operatorname{IgG}(+)$ ] with a male/female ratio of 1:1.75. In 2015, Matsuda et al (7) detected the MOG antibody in 70 patients with $\mathrm{ON}$, and the results indicated that 18 cases were MOG IgG (+), including 7 males and 11 females (1:1.57), and the MOG $\operatorname{IgG}(+)$ rate was $25.7 \%$. The above results were similar to those of the present study.

In the present study, the age at onset, course of disease, onset frequency and other immune indexes of MON IgG (+) RON patients were not significantly different compared with those of AQP4 IgG (+) RON and MON/AQP4 IgG (-) RON patients. However, another study indicated that compared with AQP4 IgG (+) patients, MOG IgG (+) patients were younger at onset, and the prevalence was not higher in females as in the present study (8). Other studies have reported that the age at onset was not significantly different between MOG IgG (+) and AQP4 IgG (+) patients (9). In 2015, Nakajima et al (10) detected the MOG antibody in patients with idiopathic ON and determined that MOG $\operatorname{IgG}(+)$ patients with idiopathic ON present with eye pain. In this study, 63.6\% patients in the MOG IgG (+) group had eye pain, but there was no significant difference compared with the AQP4 IgG (+) and MON/AQP4 IgG (-) groups. In 2014 , Sato et al (11) detected that 16 out of 215 NMO-ON patients had a MOG IgG (+) status. It was revealed that those MOG IgG (+) patients had a monophase course compared with AQP4 IgG (+) patients (11). Furthermore, in this study, the onset frequency was not significantly different between the MOG IgG (+) group and the AQP4 IgG (+) group, which may be due to the fact that all patients in the experiment had a relapsing course, and there was a lack of research on the monophase course.

Through imaging analysis, intraorbital segment involvement of RON was identified in each group of the present study. Compared with those in the MOG IgG (+) group and MOG/AQP4 IgG (-) group, more canal segment and intracranial segment involvement was observed in the AQP4 IgG (+) group. Compared with the MOG/AQP4 IgG (-) group, the optic chiasma was more frequently involved in the AQP4 IgG (+) group. Thus, the involved part of the optic nerve in MOG $\operatorname{IgG}(+)$ RON was more forward, but that in the AQP4 IgG (+) RON was more backward, which was similar to the results of Ramanathan et al (12).

Two protocols were adopted in the present study for the assessment of the worst vision at onset and visual function after recovery. First, the visual changes were directly observed, and the worst vision after onset and better vision after recovery were evaluated with 0.1 as the threshold. Second, the VFSS score was given for the degree of visual impairment prior to and after treatment, and the changes in score were observed.

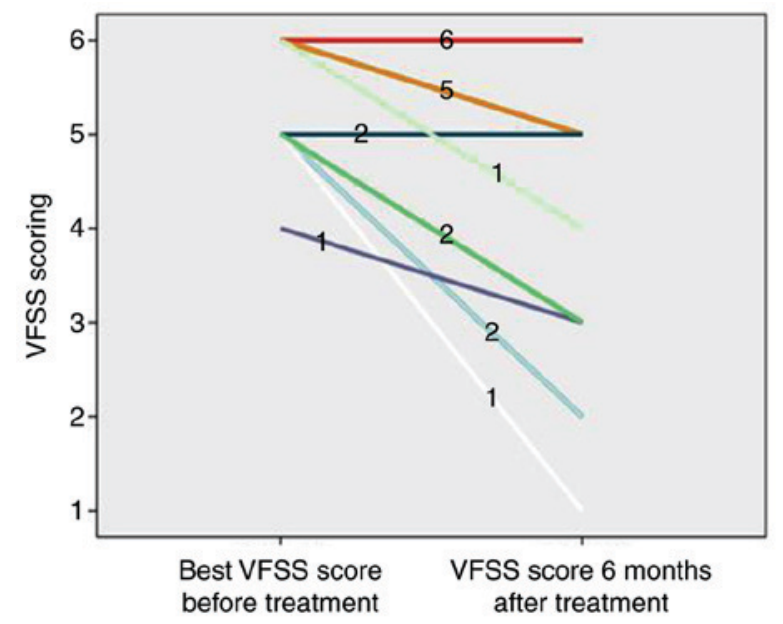

Figure 6. The VFSS score of aquaporin-4 antibody (+) recurrent optic neuritis patients prior to and at 6 months after treatment. VFSS, videofluoroscopic swallowing study.

Regardless of the evaluation protocol, the vision at onset of MOG IgG (+) RON patients was not as poor as that of AQP4 IgG (+) RON patients, and the visual recovery was better in MOG IgG (+) RON patients after hormone with/without immunosuppressive therapy. A number of studies have reported similar results (13-15).

In the present study, two cases met the diagnostic criteria for NMO-ON, including 1 case of AQP4 antibody-negative NMO-ON with RON, acute myelitis, postrema syndrome and diencephalic syndrome, which also suggested that MOG may be a potential biomarker for AQP4 IgG (-) NMO-ON. Therefore, for clinical patients with suspected NMO-ON with AQP4 IgG (-) status, the detection of serum MOG antibody may be performed, so as to facilitate early diagnosis and treatment, and thereby improve their prognosis.

Compared with the AQP IgG (+) group, the higher prevalence in females in the MOG $\operatorname{IgG}(+)$ RON group was less pronounced, the vision at onset was better, the recovery of visual function after treatment was better, and the involved part of the optic nerve was more forward. MOG IgG may be present in the serum of NMO-ON patients, which may be utilized as a potential biomarker for NMO-ON with AQP4 IgG (-) status.

\section{Acknowledgements}

Not applicable.

\section{Funding}

No funding was received.

\section{Availability of data and materials}

The datasets used and/or analyzed during the current study are available from the corresponding author on reasonable request.

\section{Authors' contributions}

JW and YP designed the present study; LL and YZ collected the data, YZ and JW analysed the data; YP, ZQ and KF 
performed the experiments, and prepared the manuscript. All authors read and approved the final manuscript.

\section{Ethics approval and consent to participate}

The present study was approved by the ethics committee of Beijing Tongren Hospital (Beijing, China). Written informed consent was obtained from the patients and/or their guardians, as well as the healthy individuals.

\section{Consent for publication}

Written informed consent was obtained from the patients and/or their guardians, as well as the healthy individuals.

\section{Competing interests}

The authors declare that they have no competing interests.

\section{References}

1. Schneider E, Zimmermann H, Oberwahrenbrock T, Kaufhold F, Kadas EM, Petzold A, Bilger F, Borisow N, Jarius S, Wildemann B, et al: Optical coherence tomography reveals distinct patterns of retinal damage in neuromyelitis optica and multiple sclerosis. Plos One 8: e66151, 2013.

2. Wan H, He H, Zhang F, Sha Y and Tian G: Diffusion-weighted imaging helps differentiate multiple sclerosis and neuromyelitis optica-related acute optic neuritis. J Magn Reson Imaging 45: 1780-1785, 2017.

3. Tan CT, Mao Z, Qiu W, Hu X, Wingerchuk DM and Weinshenker BG: International consensus diagnostic criteria for neuromyelitis optica spectrum disorders. Neurology 86: 491-492, 2016.

4. Kitley J, Waters P, Woodhall M, Leite MI, Murchison A, George J, Kuker W, Chandratre S, Vincent A and Palace J: Neuromyelitis optica spectrum disorders with aquaporin-4 and myelin-oligodendrocyte glycoprotein antibodies: A comparative study. Jama Neurol 71: 276-283, 2014.

5. Giacoppo S, Soundara RT, Galuppo M, Pollastro F, Grassi G, Bramanti P and Mazzon E: Purified Cannabidiol, the main non-psychotropic component of Cannabis sativa, alone, counteracts neuronal apoptosis in experimental multiple sclerosis. Eur Rev Med Pharmacol Sci 19: 4906-4919, 2015.
6. Saadoun S, Waters P, Owens GP, Bennett JL, Vincent A and Papadopoulos MC. Neuromyelitis optica MOG-IgG causes reversible lesions in mouse brain. Acta Neuropathol Commun 2: 35, 2014.

7. Matsuda R, Kezuka T, Umazume A, Okunuki Y, Goto H and Tanaka K: Clinical profile of Anti-Myelin oligodendrocyte glycoprotein antibody seropositive cases of optic neuritis. Neuroophthalmology 39: 213-219, 2015.

8. Hoftberger R, Sepulveda M, Armangue T, Blanco Y, Rostasy K, Calvo AC, Olascoaga J, Ramio-Torrenta L, Reindl M, Benito-Leon J, et al: Antibodies to MOG and AQP4 in adults with neuromyelitis optica and suspected limited forms of the disease. Mult Scler 21: 866-874, 2015.

9. Kim SM, Woodhall MR, Kim JS, Kim SJ, Park KS, Vincent A, Lee KW and Waters P: Antibodies to MOG in adults with inflammatory demyelinating disease of the CNS. Neurol Neuroimmunol Neuroinflamm 2: e163, 2015.

10. Nakajima H, Motomura M, Tanaka K, Fujikawa A, Nakata R, Maeda Y, Shima T, Mukaino A, Yoshimura S, Miyazaki T, et al: Antibodies to myelin oligodendrocyte glycoprotein in idiopathic optic neuritis. BMJ Open 5: e7766, 2015.

11. Sato DK, Callegaro D, Lana-Peixoto MA, Waters PJ, de Haidar JF, Takahashi T, Nakashima I, Apostolos-Pereira SL, Talim N, Simm RF, et al: Distinction between MOG antibody-positive and AQP4 antibody-positive NMO spectrum disorders. Neurology 82: 474-481, 2014.

12. Ramanathan S, Prelog K, Barnes EH, Tantsis EM, Reddel SW, Henderson AP, Vucic S, Gorman MP, Benson LA, Alper G, et al: Radiological differentiation of optic neuritis with myelin oligodendrocyte glycoprotein antibodies, aquaporin-4 antibodies, and multiple sclerosis. Mult Scler 22: 470-482, 2016.

13. Akaishi T, Nakashima I, Takeshita T, Kaneko K, Mugikura S, Sato DK, Takahashi T, Nakazawa T, Aoki M and Fujihara K: Different etiologies and prognoses of optic neuritis in demyelinating diseases. J Neuroimmunol 299: 152-157, 2016.

14. Siritho S, Sato DK, Kaneko K, Fujihara K and Prayoonwiwat N: The clinical spectrum associated with myelin oligodendrocyte glycoprotein antibodies (anti-MOG-Ab) in thai patients. Mult Scler 22: 964-968, 2016.

15. Martinez-Hernandez E, Sepulveda M, Rostasy K, Hoftberger R, Graus F, Harvey RJ, Saiz A and Dalmau J: Antibodies to aquaporin 4, myelin-oligodendrocyte glycoprotein, and the glycine receptor $\underline{\alpha} 1$ subunit in patients with isolated optic neuritis. JAMA Neurol 72: 187-193, 2015. 